\title{
Argumentos formales y visuales en clase de geometría a nivel primaria
}

\section{Formal and Visual Arguments in Primary Geometry Class}

\author{
Jonathan Cervantes-Barraza ${ }^{1}$ \\ Guadalupe Cabañas-Sánchez ${ }^{2}$
}

\begin{abstract}
Resumen. En este artículo se presenta un estudio relacionado con los argumentos que esgrimen alumnos de primaria en la resolución de problemas geométricos que contienen una representación gráfica. En concreto, se analizaron sus argumentos mediante el modelo argumentativo de Toulmin, que permitió la reconstrucción de los mismos desde las intervenciones de los alumnos. Así, los resultados señalan que en la resolución de tareas geométricas, los estudiantes de primaria fundamentan sus argumentos a partir de características visuales del objeto matemático en estudio, desde fórmulas o propiedades pertenecientes a la matemática escolar.
\end{abstract}

Palabras clave: argumentación; argumentos visuales; argumentos formales; modelo de Toulmin.

Fecha de recepción: 4 de febrero de 2017. Fecha de aceptación: 1 de noviembre de 2017

1 Estudiante del Doctorado en Ciencias con Especialidad en Matemática Educativa, CIMATE Universidad Autónoma de Guerrero UAGro, México, jacbmath@hotmail.com.

2 Doctora en Ciencias con Especialidad Matemática Educativa en el Departamento de Matemática Educativa del Centro de Investigación y Estudios Avanzados del IPN, profesora titular CIMATE Universidad Autónoma de Guerrero UAGro, México, gcabanas.sanchez@gmail.com. 


\begin{abstract}
In this article we present a study related to the arguments made by elementary school students in the resolution of geometric problems that contain a graphic representation. In particular, their arguments were analyzed through the argumentative model of Toulmin, which allowed its reconstruction from the interventions made by them. Thus, the results indicate that in the resolution of geometric tasks, elementary school students base their arguments on visual characteristics of the mathematical object under study, from formulas or properties belonging to school mathematics.
\end{abstract}

Keywords: Argumentation; visual arguments; formal arguments; Toulmin model.

\title{
1. CONTEXTUALIZACIÓN Y PREGUNTA DE INVESTIGACIÓN
}

La argumentación matemática es actualmente uno de los centros de interés en Didáctica de las Matemáticas (Solar, Azcárate y Deulofeu, 2011) y se ha convertido en una línea de investigación relevante en el campo de la Educación Matemática, en la cual el análisis de la reconstrucción y evaluación de argumentos en la clase de matemáticas ha ganado importancia (Inglis y Mejia-Ramos, 2005, 2007, 2009; Krummheuer, 1995, 2007, 2015; Whitenack y Knipping, 2002; Yackel, 2002). Asimismo, entidades que realizan estudios para la mejora de la práctica docente, por ejemplo el Nacional Council of Teachers of Mathematics (NCTM), propusieron como uno de los objetivos de la Educación Matemática ayudar a los estudiantes para que aprendan a participar en la producción de argumentos matemáticos, consolidando la argumentación como una oportunidad de aprendizaje disponible desde el inicio de la escolarización y no solamente reservada para estudios avanzados (NCTM, 2000). Con base en lo anterior, sobresalen dos aspectos importantes sobre los cuales esta investigación se centra: el primero se refiere a la promoción de argumentos entre los alumnos a partir de tareas matemáticas y el segundo, promover la argumentación matemática en estudiantes de primaria.

Tomando en cuenta la argumentación matemática como un proceso del discurso social dinámico que permite descubrir nuevas ideas matemáticas y convencer a otros sobre la validez de una afirmación (Rumsey y Langrall, 2016), la argumentación se convierte en una oportunidad de aprendizaje para los 
estudiantes a la hora de resolver tareas donde ésta es el medio para socializar procedimientos, respuestas y puntos de vista. De esta manera, argumentación y discurso en el sentido de Rumsey y Langrall (2016) son componentes del desarrollo de la matemática como dominio que, a su vez, permite la construcción de argumentos viables y la crítica del razonamiento de los demás.

El hecho de investigar los argumentos de los alumnos en clase de matemáticas de nivel primaria dentro del contexto de tareas geométricas, está relacionado con la hipótesis de investigación: sus argumentos en el contexto de problemas geométricos no están fundamentados en la matemática formal estudiada, es decir, no recurren a definiciones ni fórmulas matemáticas en el momento de presentar los resultados. Por tanto, se generó la pregunta de investigación, planteada de la siguiente manera: ¿Cuáles son los argumentos de los estudiantes cuando resuelven problemas geométricos que incluyen una representación gráfica?

\section{SUSTENTO TEÓRICO}

El presente reporte de investigación recae en conceptos de la teoría argumentativa de Toulmin (2003), presentados de forma general y haciendo hincapié en qué se entiende por argumentación, argumento y cada una de sus partes.

\subsection{ARGUMENTACIÓN MATEMÁtiCA EN EL SALÓN DE CLASE}

El término argumentación ha sido implementado por investigadores en el campo de la Educación Matemática (Walton, 2009; Potari, Zachariades y Zaslavsky, 2010) en el sentido de Toulmin (2003); en esta investigación se implementó el término argumentación en el mismo sentido, de manera que se entenderá como toda la actividad central de establecer aserciones, refutar, soportar la producción de razones y hacer críticas sobre esas razones. Asimismo, se concibe un argumento en el sentido de cadenas de razonamientos, como secuencia conectada de aserciones y razones que conforman el contenido y la fuerza de la posición del argumentador (Toulmin, 2003).

Para Toulmin, Rieke y Janik (1984) los argumentos tienen una estructura conformada por seis elementos: el dato es la información empleada para establecer una aserción o conclusión, la aserción es la tesis o afirmación que crea el argumentador y está sustentada por la garantía, cuyo papel es conectar los 
datos con la aserción; además, se desprende de la garantía el respaldo, que tiene como función soportarla, pues presenta un apoyo teórico: ejemplos o casos muy generales, el calificador modal evidencia la fuerza del argumento expresado en frases como siempre que, certeramente, para todo, algunas veces, entre otros; por último, la refutación presenta las excepciones de la aserción, las cuales no pueden ser sostenidas por la garantía del argumento (Ver Figura 1).

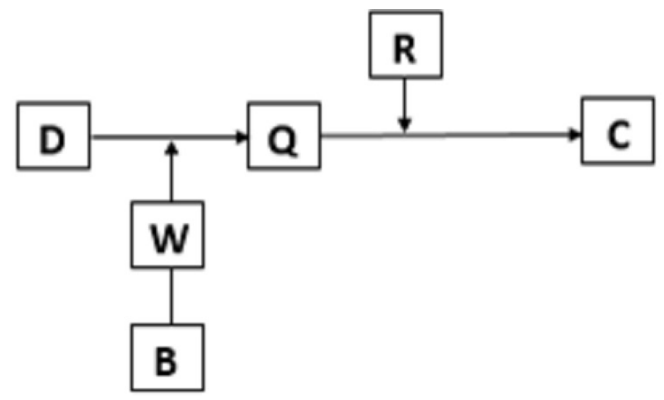

Figura 1. Modelo argumentativo de Toulmin (Inglis y Mejia-Ramos, 2005).

Tal es el caso de investigaciones desarrolladas por Goizueta y Planas (2013); Solar, Azcárate y Deulofeu (2011), la argumentación es concebida como el acto de producir razones, una habilidad básica desarrollada de manera progresiva a lo largo de las etapas de la educación obligatoria. Al argumento lo definen como una razón o razones ofrecidas a favor o en contra de una proposición. Con base en lo anterior, es importante remarcar una distinción, debido a que un argumento refiere a la lista de razones conectadas entre sí para generar la argumentación, en cambio la argumentación es el proceso que incluye todas las acciones realizadas (por ejemplo identificar datos, presentar aserciones, establecer garantías, refutar, responder a las refutaciones).

Por su parte, Solar, Azcárate y Deulofeu (2011) realizaron su investigación sobre argumentación en clase de matemáticas y al respecto señalan que en el aula no es frecuente encontrar discusiones donde aparezcan todos los procesos ${ }^{3}$ de la argumentación. De esta manera, proponen como condición básica para que exista argumentación, la presencia de al menos cuatro procesos: datos,

\footnotetext{
3 Los procesos, de acuerdo con Solar, Azcárate y Deulofeu (2011), se refieren a las partes de un argumento Toulmin (2003).
} 
conclusión, garantía y refutación; este cuarto proceso es uno de los más relevantes, puesto que evidencia posturas diferentes entre los estudiantes.

El término refutación es tomado en el sentido de Toulmin (2003), quien lo define como la oportunidad de especificar las excepciones de la conclusión/ aserción de un argumento. Asimismo, una refutación puede ofrecerse en el contexto de una discusión, y en su garantía incluir un contraejemplo u otras circunstancias en las que el resto del argumento tendría que ser modificado, obligando a cambiar el tipo de razonamiento (inductivo, deductivo, abductivo o por analogía) subyacente en los argumentos de los participantes (Conner, Singletar, Smith, Wagner y Francisco, 2014: 190).

Por otro lado, investigadores han centrado su atención en el estudio de la refutación dentro de las prácticas argumentativas; Reid, Knipping y Crosby (2011) reconocen tres formas de refutar un proceso argumentativo: a) Los datos del argumento son refutados y se deja la conclusión en duda, b) Se refuta la garantía y se deja la conclusión en duda y c) La conclusión puede ser refutada, lo cual implica la invalidez de los datos o de la garantía, aunque no se tenga clara la validez de unos u otra.

\subsection{TIPOS DE ARGUMENTOS}

La definición de los tipos de argumentos presentados en este reporte fue seleccionada de investigaciones relacionadas con su estudio (Viholainen, 2008; Inglis y Mejía-Ramos, 2009; Groarke, 2009). Los tipos de argumentos fueron identificados con base en las garantías expuestas por los estudiantes en los resultados de la tarea matemática, para luego seleccionar entre diferentes definiciones, de modo que se optó por la clasificación de los argumentos en visuales y formales.

\subsubsection{Argumentos visuales}

Se caracterizan por presentar garantías que fundamentan aserciones informales de conceptos o situaciones (Viholainen, 2008). Así, en un argumento informal se identifican solo características físicas o visuales sin presentar definiciones, teoremas o fórmulas pertenecientes a la matemática. Los argumentos visuales son más que premisas/conclusiones expresadas en su totalidad o parcialmente por medios visuales (Groarke, 2009: 230). Desde estas perspectivas, un argumento visual es aquel que está fundamentado (por ejemplo, la garantía) en 
características visuales basadas en las representaciones de los objetos matemáticos ("el área del triángulo $\mathrm{ABC}$ es mayor que el área del triángulo MNP, porque se ve más grande en el dibujo").

Desde la postura de Inglis y Mejía-Ramos (2009: 100), los argumentos visuales son aquellos que se basan en imágenes e incluyen no solamente las cifras extraídas o generadas por ordenador y diagramas, sino también imágenes mentales en la práctica matemática. Este punto de vista es más específico en términos de la visualización de los objetos matemáticos, debido a que el estudiante considera la imagen mental o representación del objeto como un sustento para argumentar.

Por otra parte, Groarke (2009) estableció 5 tesis que permiten estudiar los argumentos visuales. Tesis 1: Los datos y conclusiones se pueden transmitir con medios visuales, Tesis 2: Una garantía puede expresarse visualmente como conexión entre los elementos datos-aserción, Tesis 3: Los calificadores modales pueden ser expresados visualmente como situaciones de incertidumbre, Tesis 4: El respaldo de un argumento puede ser transmitido visualmente, y Tesis 5: El modelo de Toulmin tiene deficiencias en el contexto del argumento visual. En esta investigación se implementaron las dos primeras tesis, ya que tienen como función identificar y explicar las situaciones en las cuales los estudiantes retoman elementos de la argumentación (datos, garantías o aserción) basados en características del objeto matemático (tamaño, forma, inclinación), con el fin de establecer y fundamentar sus argumentos.

\subsubsection{Argumentos formales}

Al estudiar los argumentos formales se debe reconocer la función de la garantía en la estructura argumentativa, pues brinda una conexión entre los datos y la aserción, con el fin de soportar la aserción o conclusión del argumentador (Toulmin et al., 1984). Así, la garantía cumple un rol importante en los argumentos establecidos por los estudiantes, dado que permite, a la vez, su clasificación en formales o visuales.

Los argumentos formales, desde el punto de vista teórico de Viholainen (2008), son clasificados de acuerdo al contenido de la garantía, dado que este elemento argumentativo establece la conexión entre dos partes esenciales de cualquier argumento: los datos y la aserción. Más aún, Viholainen (2008) concibe un argumento como formal cuando su garantía se basa puramente en los elementos del sistema axiomático formal de las matemáticas. En otras palabras, un argumento 
es formal si muestra explícitamente la manera como se fundamenta lógicamente en las definiciones, axiomas, teoremas y fórmulas matemáticas. En contraste, un argumento visual presenta una garantía en la que se incluyen características del objeto matemático (tamaño, medidas o casos particulares) para sustentarlo y, al presentar estas garantías, se deja de lado la matemática formal, lo cual no lo hace un argumento inválido, aunque sí un argumento carente de fundamentos matemáticos.

\section{METODOLOGÍA DE LA INVESTIGACIÓN}

Esta investigación se basó en el paradigma de investigación de diseño (Steffe y Thompson, 2000; Molina, Castro, Molina y Castro, 2012), cuya naturaleza es primordialmente cualitativa, y de forma puntual enmarca dentro de éste, lo que se denomina Experimento de Enseñanza (Teaching Experiment). En la postura de Steffe y Thompson (2000: 273) un experimento de enseñanza consiste en una secuencia de episodios de clase que incluye un agente de enseñanza, uno o más estudiantes, un testigo de los episodios y un método de grabación de lo que sucede durante el episodio. Así, esta investigación se centra en el estudio de los argumentos producidos por alumnos de primaria en el contexto de un experimento de enseñanza, el cual tiene como objetivo experimentar de primera mano el aprendizaje y razonamiento matemático de los estudiantes por parte de los investigadores (Steffe y Thompson, 2000).

El experimento estuvo conformado por dos sesiones video grabadas de 45 minutos cada una. En la primera, el profesor investigador interactuó con los alumnos mediante preguntas relacionadas con las formas de calcular el área de cuadrados y triángulos. En la segunda sesión, los estudiantes desarrollaron la tarea matemática en dos momentos, primero de forma individual sobre su hoja de trabajo, y luego de forma colectiva compartieron sus respuestas con toda la clase.

\subsection{PARTICIPANTES Y TAREA MATEMÁTICA}

El Experimento de Enseñanza se llevó a cabo con la participación de seis estudiantes mexicanos que cursaban el último grado de educación primaria $\left(6^{\text {th }}\right)$, el rango de edades oscilaba entre 10 y 12 años. Básicamente, el experimento consistió en la aplicación de una tarea matemática cuyo propósito era generar 
la argumentación en el salón de clases. Esta tarea fue desarrollada por el investigador, quien tomó el rol de profesor ante el grupo para conducir a los estudiantes y que argumentaran sus respuestas.

El diseño de la tarea matemática planteada fue validado por el grupo de investigación en argumentación matemática al que pertenecen los autores de este reporte, quienes hicieron aplicaciones piloto para validarla. La tarea se centró en el estudio de la conservación del área de triángulos construidos sobre una cuadrícula. El tema fue tomado con base en el programa de estudios propuesto por la Secretaría de Educación Pública (SEP) de México (ver Figura 2).

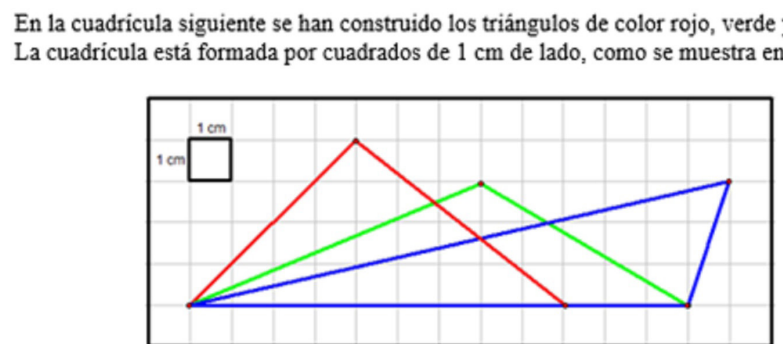

Analiza y responde ¿qué triángulo tiene mayor área?, argumenta tu respuesta seleccionada.

a) El área del triángulo rojo.

b) El área del triảngulo verde.

c) El área del triángulo azul.

d) Ninguno, todos tienen la misma área.

¿Por qué?

Figura 2. Tarea matemática propuesta (autores).

\subsection{ANÁLISIS DE LA ARGUMENTACIÓN}

El análisis de la escena de clase se llevó a cabo durante varias etapas, en la primera hicimos la transcripción del video sobre una matriz con tres columnas: el número del diálogo, nombre del participante y texto transcrito (ver Anexo). La segunda etapa consistió en el análisis del contenido de los argumentos estudiantiles con base en la reconstrucción de la argumentación (Toulmin, 2003) y, por último, en la tercera etapa se contrastó el análisis de los contenidos de los argumentos con la teoría, para obtener la respuesta a la problemática planteada. 
El análisis de los argumentos de los alumnos en la tarea matemática dentro del salón de clase fue realizado con base en el modelo propuesto por Toulmin (2003), aunque específicamente para analizar los argumentos de tipo formal tomamos como referencia las ideas de Viholainen (2008). Metodológicamente, se identificaron los elementos del núcleo de los argumentos (datos, aserción, garantía); de manera similar, varios autores (Conner, Singletary, Smith, Wagner y Francisco, 2014; Krummheuer, 1995, 2007; Whitenack y Knipping, 2002) han encontrado resultados sobre la estructura argumentativa. Además de identificar el núcleo, en esta investigación se reconoció la refutación de los estudiantes en la estructura argumentativa, junto con la participación del profesor.

\subsection{ALgUNAS CONVENCIONES DEL MODELO DE TOULMIN}

Sobre la estructura argumentativa establecida por Toulmin, planteamos ciertas convenciones para facilitar la identificación de los elementos del argumento y los comentarios del profesor durante la transcripción-reconstrucción de la argumentación. Estas convenciones tienen que ver con representar los datos (D) mediante un rectángulo, la garantía (W) con un óvalo, la aserción (C) con un rectángulo de esquinas curvas, el respaldo con un óvalo con línea gruesa y, por último, la refutación (R) con un rombo, además de que los comentarios del docente se representan con globos de diálogos. Dentro de cada elemento de la argumentación se indica el nombre del participante con negritas y el texto transcrito del video. Estas convenciones se muestran en la sección de resultados de la investigación, con el objetivo de facilitar la interpretación de lo sucedido en el salón de clase (ver Figura 3).

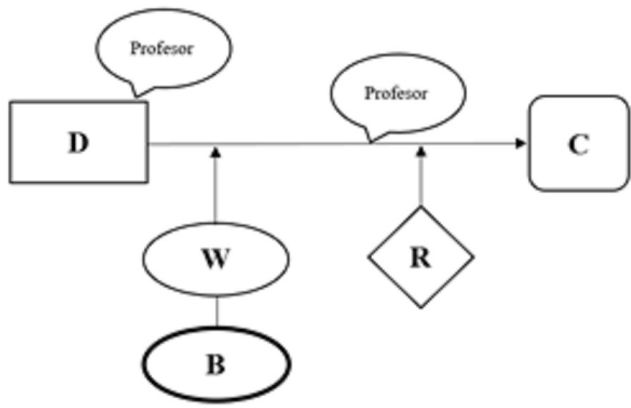

Figura 3. Convenciones del modelo argumentativo de Toulmin (autores). 


\section{RESULTADOS}

Los resultados se presentan en términos de los argumentos esgrimidos por los alumnos en la resolución de la tarea matemática propuesta. Emergieron luego de que el profesor preguntara: ¿qué triángulo tiene mayor área?, para así gestionar la argumentación desde las respuestas de los estudiantes frente al grupo de compañeros.

El primer argumento corresponde al estudiante Sam (ver Figura 4), cuya respuesta fue: todos tienen la misma área. Aserción fundamentada en datos tomados de la tarea y sustentada en el número de cuadros que tienen los triángulos, tanto en la base como en la altura; este hecho se identifica con la Tesis 1, la cual establece la toma de los datos y/o aserción desde lo visual por parte del alumno. Además, en su garantía implementó la cantidad de cuadrados pertenecientes en la base y altura de los triángulos, para luego aplicar la fórmula del área de triángulos, que se considera el respaldo del argumento.

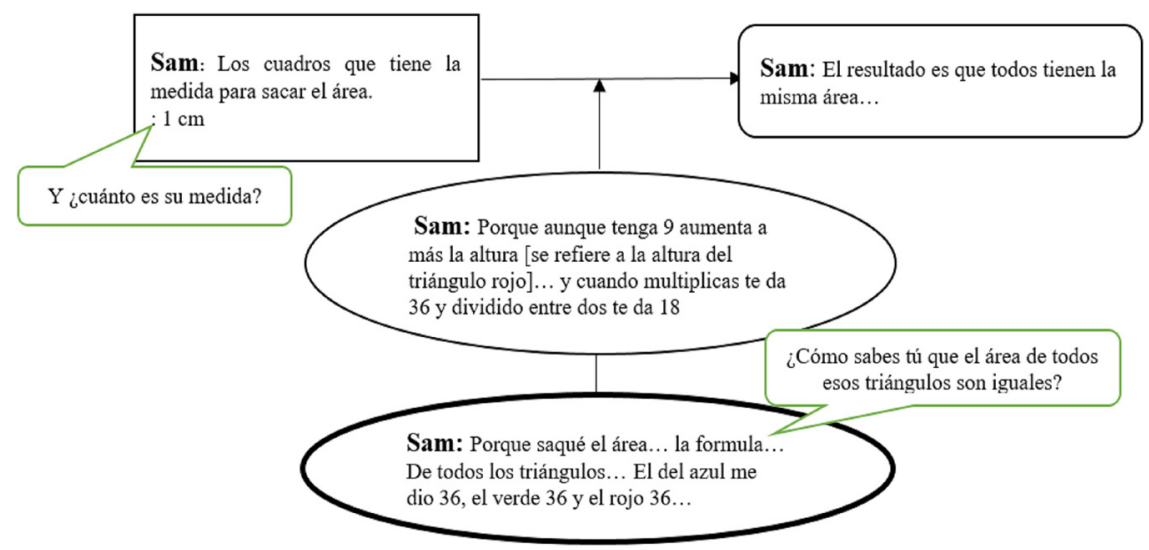

Figura 4. Argumento de Samuel.

Como se puede apreciar, en el argumento de Sam, el profesor -mediante preguntas- hace que emerjan la garantía y el respaldo, los cuales están fundamentados en la fórmula del área del triángulo, un teorema ${ }^{4}$ que forma parte de la matemática impartida en este grado escolar, y esto permite clasificar el argumento de Sam como formal.

\footnotetext{
4 La fórmula del área de triángulos es un teorema según Clements, O’Daffer y Cooney (1998).
} 
En el argumento de Erik (ver Figura 5), quien también asevera: todos tienen la misma área, esgrime uno fundamentado en datos tomados del enunciado del problema (Tesis 1). Así también, en su argumento de garantía evidencia la comparación de la cantidad de cuadros que tiene la altura y base de todos los triángulos, para luego recurrir a la fórmula del área del triángulo.

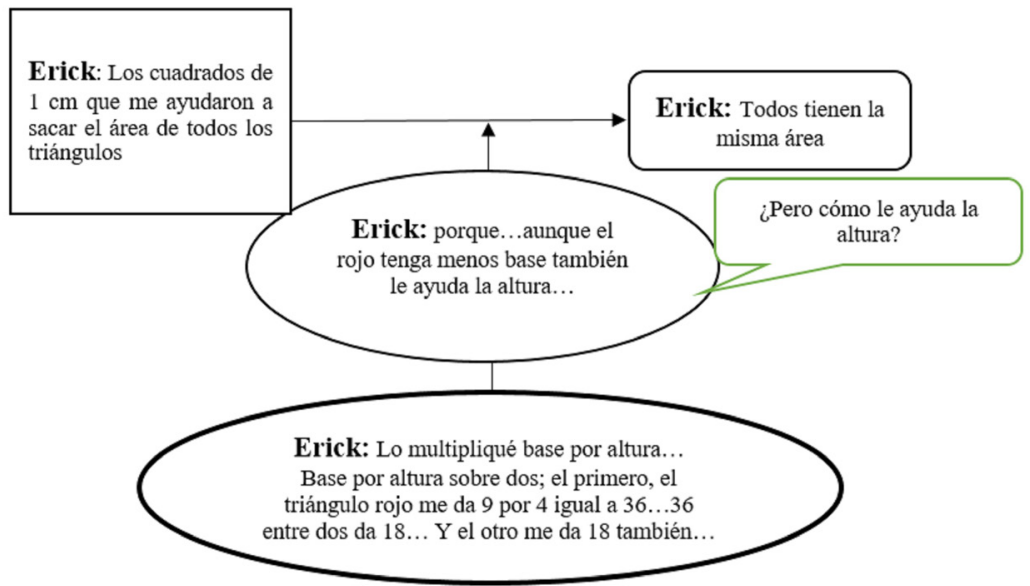

Figura 5. Argumento de Erick.

El argumento de Erick es de tipo formal porque la garantía y el respaldo hacen alusión a la fórmula del área del triángulo, que pertenece a la formalidad de la matemática, de manera que Erick no sustentó su argumento desde características visuales de la representación del triángulo, sino a partir de fundamentos matemáticos.

El argumento de Víctor (ver Figura 6) identificó los datos (Tesis 1) desde la tarea, posteriormente presentó su aserción: el área del triángulo azul. Para conectar los datos con la aserción establece un argumento de garantía que describe la medida de la base y su respectiva altura. Esta garantía no remite a la formalidad matemática, más bien basó su garantía en lo visual para conectar los datos con la aserción (Tesis 2), lo cual permite clasificar el argumento de este estudiante como de tipo visual y sin respaldo. 


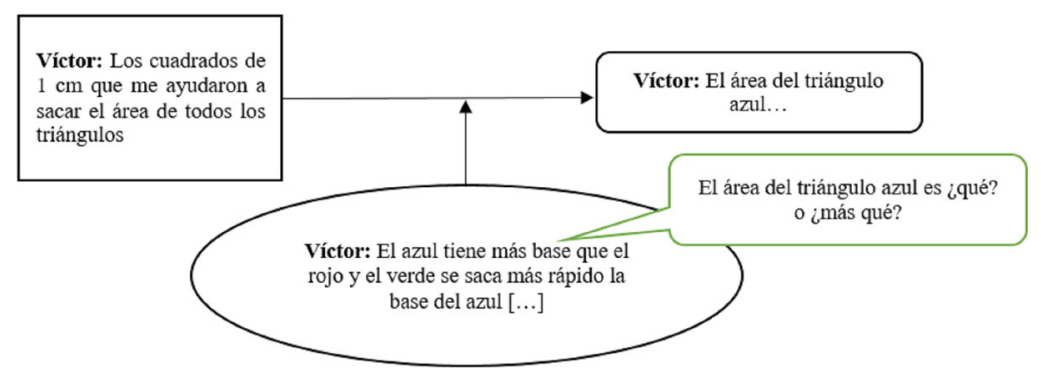

Figura 6. Argumento de Víctor.

Luego de haber analizado los argumentos de los alumnos, se presentan argumentos donde se puede observar un elemento clave en la argumentación, como es la refutación. En estos casos los estudiantes presentan sus puntos de vista luego de escuchar a otro compañero.

Veamos el argumento de Álvaro (ver Figura 7), quien tomó los cuadros de 1 cm cuadrado (Tesis 1) para establecer la aserción: todos los triángulos tienen la misma área. Este alumno justifica la conexión entre datos y aserción o presenta su argumento de garantía mediante la fórmula del área de triángulos, por tanto, su argumento es de tipo formal. Por otro lado Víctor, quien había expresado su argumento (Figura 6), refuta la respuesta de Álvaro (Figura 7), en particular refuta directamente la aserción, y en la garantía de su refutación incluye características visuales presentes en su argumento. Lo anterior conllevó a que el argumento de Álvaro no fuera modificado o puesto en tela de juicio, como lo indican Conner, Singletar, Smith, Wagner y Francisco (2014: 190).

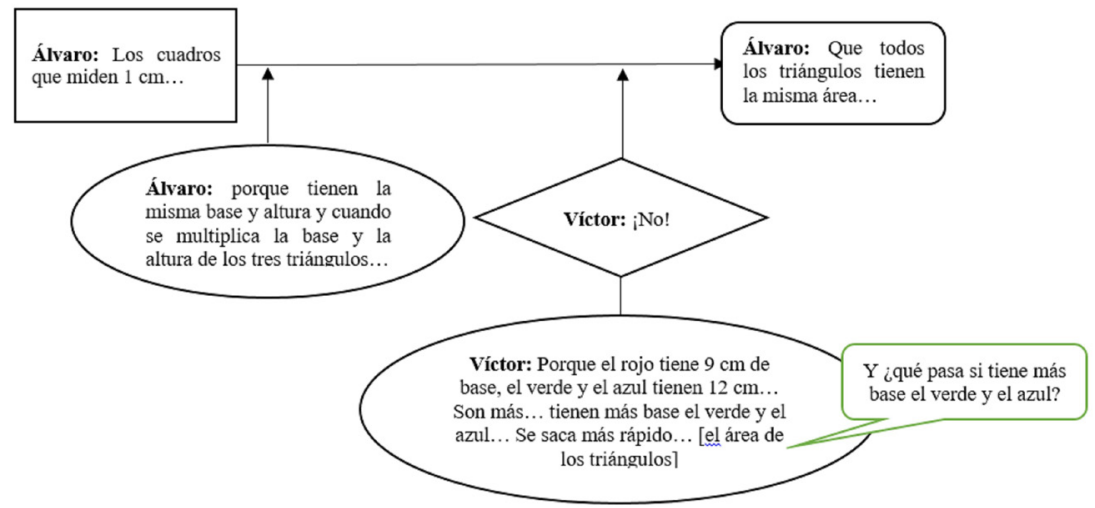

Figura 7. Argumento de Álvaro. 
Asimismo, en el argumento de Ingrid (ver Figura 8) puede apreciarse que los datos considerados para sustentar la aserción y establecer su garantía fueron los cuadros de la cuadrícula (Tesis1), y luego estableció la aserción: el triángulo azul. Esta conexión entre datos y aserción la fundamentó en un rasgo visual (Tesis 2): "el azul se ve más grande", lo cual indica que tuvo en cuenta el tamaño de los tres triángulos como garantía, por tanto, este argumento es de tipo visual. Enseguida, la refutación de Víctor: no importa la forma sino el resultado de multiplicar la base por la altura y dividirla por dos, evidenció la falta de una garantía enmarcada dentro de la matemática enseñada. Además, como se puede apreciar en el esquema, Álvaro también refutó el argumento de Ingrid y apoyó la garantía al expresar: no importa el tamaño del triángulo sino el resultado de aplicar la fórmula.

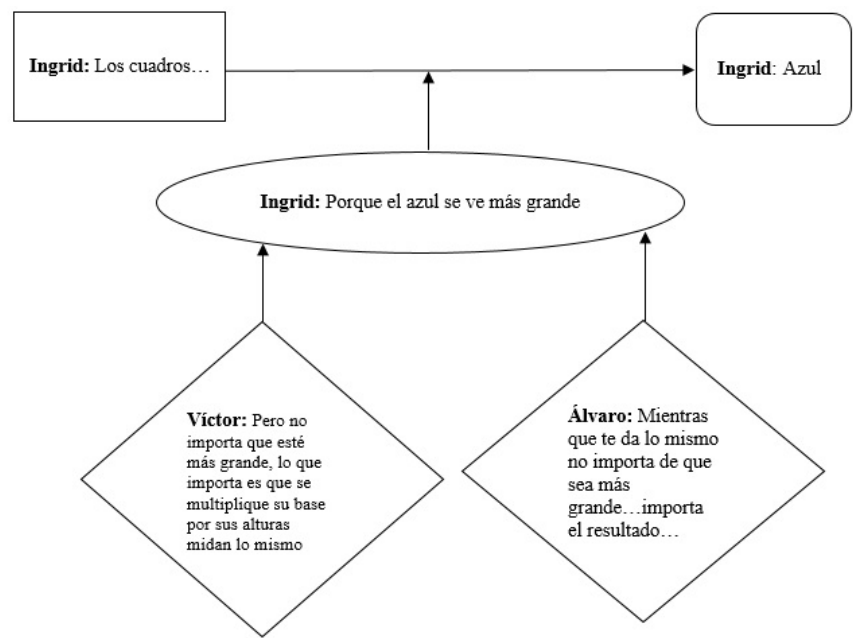

Figura 8. Argumento de Ingrid.

Al ser refutado el argumento de Ingrid, se evidencia la ausencia de una garantía relacionada con la matemática, mientras que Víctor y Álvaro presentaron sus diferentes puntos de vista con la intención de comunicarle a Ingrid la falta de un sustento a su argumento, despreciando la forma o el tamaño del triángulo (lo visual).

Por último, en el argumento de Itzel (ver Figura 9) para establecer la aserción: el triángulo azul, seleccionó como datos los cuadros de la cuadrícula (Tesis 1) y fundamentó su argumento en la cantidad de cuadros que tiene cada triángulo en sus bases. Con base en el contenido de la garantía, este argumento es 
clasificado como visual debido a que Itzel tuvo en cuenta que a mayor cantidad de cuadros en la base de los triángulos, estos tendrán mayor área (Tesis 2: tomar características visuales para fundamentar el argumento). Álvaro también refutó el argumento de Itzel, específicamente rechazó la medida de la base del triángulo como garantía, y con ello cambió el foco de atención sobre la base, hacia el resultado de la fórmula del área de triángulos.

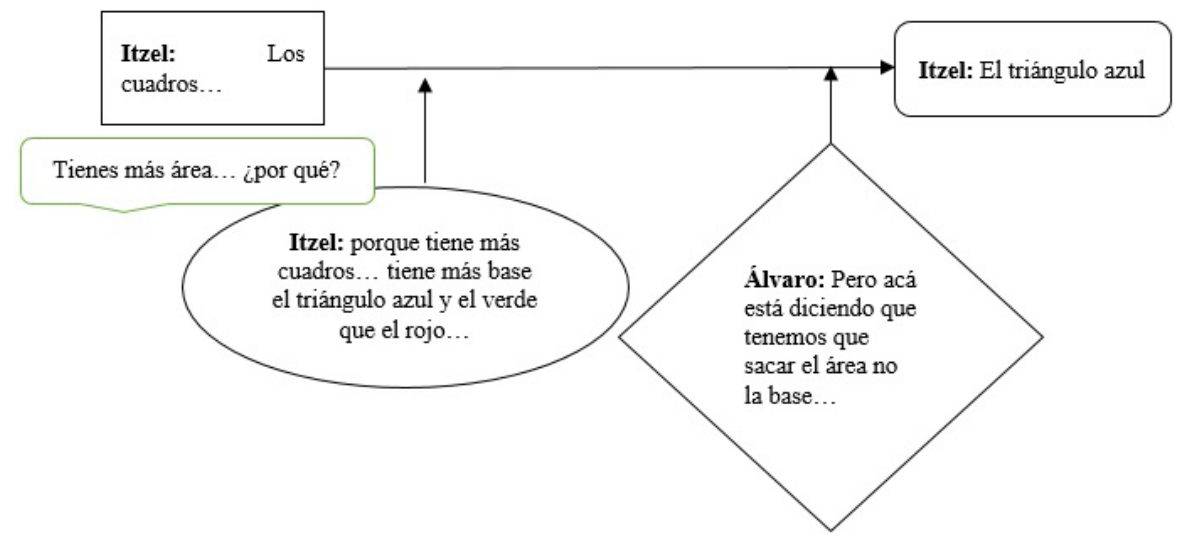

Figura 9. Argumento de Itzel.

Es pertinente subrayar cómo la ausencia de un soporte matemático hace que el argumento de Itzel fuera refutado en la clase de matemáticas, dado que su argumento es de tipo visual, fundamentado en rasgos visuales (cantidad de cuadros de la base de los triángulos) y su garantía no hace alusión a la matemática formal como sustento.

\section{DISCUSIÓN Y CONCLUSIONES}

En esta sección se presenta un contraste entre los resultados de este y otros estudios donde se ha empleado el modelo de Toulmin para analizar la argumentación en el nivel de educación básica primaria. Específicamente, Zacharos, Pournatzi, Renzos y Shiakalli (2016) estudiaron las formas de los argumentos empleados por niños de educación preescolar y las habilidades que poseen para establecer argumentos enmarcados en el razonamiento lógico. En su estudio, los resultados señalan que la mayoría de las estructuras argumentativas de los alumnos de 
preescolar estudiados se fundamentan en la lógica formal, incluyendo el razonamiento inductivo a la hora de establecer diálogos y argumentos.

No obstante que el objetivo de la investigación presentada en este reporte no era identificar el tipo de razonamiento lógico, sino el tipo de argumento que esgrimen los estudiantes en una tarea de geometría, en esta propuesta se identificaron similitudes con la investigación realizada por Zacharos et al. (2016), en vista de que los argumentos de los estudiantes implementaron razonamientos basados en la deducción, aunque no con un nivel de formalidad (demostraciones matemáticas), sino que implementaron la fórmula para encontrar el área de triángulos y, a la vez, sustentar sus argumentos.

Por otra parte, Krummheuer (2013) estudió las estructuras de los argumentos de alumnos de tercer grado de primaria y estableció, a modo de resultado, que los argumentos empleados por ellos se basan en argumentos diagramáticos cuya garantía está fundamentada en diagramas, figuras, representaciones gráficas, y también en argumentos narrativos emitidos por los estudiantes, basándose en narraciones de eventos o secuencias de acciones implementadas para llevar a cabo su tarea matemática. En contraste con los resultados de esta investigación, en nuestro caso los argumentos diagramáticos se relacionan con los visuales, ya que describen un tipo de argumento basado en gráficas, imágenes y características del objeto matemático en estudio.

Con base en los resultados del análisis de los argumentos emitidos por los alumnos, en su mayoría fueron identificados argumentos visuales (por ejemplo Víctor, Itzel, Ingrid, y Víctor como refutación). Este tipo de argumento se caracterizó por presentar afirmaciones cuya garantía carecía de un sustento matemático, fórmula, teorema o definición. Además, en relación con las tesis 1 y 2 planteadas por Groarke (2009), es de señalarse que el estudiante puede tomar los datos, la garantía y hasta la aserción desde la representación gráfica, es decir desde lo visual.

Por ende, los argumentos visuales no son evaluados como errados en esta investigación, pero si corroboran la hipótesis de investigación, pues los estudiantes dejan de lado la formalidad de la matemática cuando expresan sus respuestas y justificaciones en el contexto de la resolución de problemas geométricos. Del mismo modo se identificaron argumentos de tipo formal que incluyen garantías fundamentadas en la fórmula para encontrar el área del triángulo, de manera implícita, puesto que de forma escrita no fue perceptible, solo enunciada por parte de los estudiantes (en el caso de Sam, Álvaro, Erick). A causa de que los alumnos emplearon argumentos visuales y formales en la tarea planteada -con predominio 
de los visuales (4 de 7)-, se recomienda proponer tareas cuyo contenido se relacione con la exploración de fundamentos matemáticos (fórmulas, propiedades, reglas), para motivar a los estudiantes a sustentar sus resultados mediante argumentos basados en garantías desde la matemática impartida en clase.

\section{REFERENCIAS}

Clements, S., O’Daffer, P. \& Cooney, T. (1998). Geometría. México. Pearson.

Conner, A., Singletary, L., Smith, A., Wagner, P. \& Francisco, P. (2014). Identifying Kinds of Reasoning in Collective Argumentation, Mathematical Thinking and Learning, 16:3, 181-200, DOI: 10.1080/10986065.2014.921131.

Goizueta, M. \& Planas, N. (2013). Temas emergentes del análisis de interpretaciones del profesorado sobre la argumentación en clase de matemáticas. Enseñanza de las Ciencias. 31(1), 61-78.

Groarke, L. (2009). Five Theses on Toulmin and Visual Argument. In: F. H. van Eemeren \& Garssen, B. (eds.). Pondering on Problems of Argumentation. Twenty Essays on Theoretical Issues. University of Amsterdam, The Netherlands.

Inglis, M., \& Mejia-Ramos, J. P. (2005). La fuerza de la aserción y el poder persuasivo en la argumentación en matemáticas. Revista EMA: Investigación e Innovación en Educación Matemática, 10, 327-352.

Inglis, M., \& Mejía-Ramos, J. P. (2009). On the Persuasiveness of Visual Arguments in Mathematics. Foundations of Science, 14(1-2), 97-110. http://doi.org/10.1007/ s10699-008-9149-4.

Inglis, M., Mejia-Ramos, J. P., \& Simpson, A. (2007). Modelling Mathematical Argumentation: The Importance of Qualification. Educational Studies in Mathematics, 66(1), 3-21. http://doi.org/10.1007/s10649-006-9059-8.

Krummheuer, G. (1995). The Ethnology of Argumentation. In: P. Cobb and H. Bauersfeld (eds.). The Emergence of Mathematical Meaning: Interaction in Classroom Cultures. Hillsdale: Erlbaum, pp. 229-269.

Krummheuer, G. (2007). Argumentation and participation in the Primary Mathematics Classroom. Two Episodes and Related Theoretical Abductions. Journal of Mathematical Behavior, 26(1), 60-82.

Krummheuer, G. (2015). Methods for Reconstructing Processes of Argumentation and Participation in Primary Mathematics Classroom Interaction. En Bikner-Knipping et al. (eds.). Approaches to Qualitative Research in Mathematics Education (pp. 51-72). New York: Advances in Mathematics Education. 
Krummheuer, G. (2013). The Relationship between Diagrammatic Argumentation and Narrative Argumentation in the Context of the Development of Mathematical Thinking in the Early Years. Educational Studies Mathematics, 84:249-265, DOI: 10.1007/ s10649-013-9471-9.

Molina, M., Castro, E., Molina, J., \& Castro, E. (2012). Un acercamiento a la investigación de diseño a través de los experimentos de enseñanza. Enseñanza de las Ciencias, 29(1), 075-088.

National Council of Teachers of Mathematics (2000). Principles and Standards for School Mathematics. Reston, VA: Author.

Potari, D., Zachariades, T. \& Zaslavsky, O. (2010). Mathematics Teachers' Reasoning for Refuting Students' Invalid Claims. In: V. Durand-Guerrier, France S. Soury-Lavergne, F. Arzarello (eds.). Proceedings of Sixth Congress of European Research in Mathematics Education, pp. 281-290, Lyon France: CERME 6.

Reid, D., Knipping, C. \& Crosby, M. (2011). Refutations and the Logic of Practice. PNA, 6(1), 1-10. Rumsey, C. \& Langrall, C. W. (2016). Promoting Mathematical Argumentation. Teaching Children Mathematics, 22(7), 413-419.

Solar, H., Azcárate, C. \& Deulofeu, J. (2011). Competencia de argumentación en, la interpretación de gráficas funcionales. Enseñanza de las Ciencias, 30(3): 133-154.

Steffe, L. P. \& Thompson, P. W. (2000). Teaching Experiment Methodology: Underlying Principles and Essential Elements. In: R. Lesh \& A. E. Kelly (eds.). Research Design in Mathematics and Science Education, pp. 267-307. Hillsdale, NJ: Erlbaum.

Toulmin, S. (1958/2003). The Uses of Argument. New York: Cambridge University Press. Toulmin, S. E., Rieke, R. D. \& Janik, A. (1984). An Introduction to Reasoning (2nd ed.). New York, London: MacMillan.

Viholainen, A. (2008). Prospective Mathematics Teachers' Informal and Formal Reasoning about the Concepts of Derivative and Differentiability. Dissertation. En: http://www. math.jyu.fi/research/reports/rep115.pdf.

Whitenack, J. W. \& Knipping, N. (2002). Argumentation, Instructional Design Ttheory and Students' Mathematical Learning: A Case for Coordinating Interpretive Lenses. Journal of Mathematical Behavior, 21(4), 441-457. http://doi.org/10.1016/ S0732-3123(02)00144-X.

Yackel, E. (2002). What We Can Learn from Analyzing the Teacher's Role in Collective Argumentation. Journal of Mathematical Behavior, 21(4), 423-440. http://doi. org/10.1016/S0732-3123(02)00143-8.

Zacharos, K., Pournantzi, V., Moutsios-Rentzos, A. \& Shiakalli, M. A. (2016). Forms of Argument Used by Pre-school Children. Educational Journal of the University of Patras. UNESCO Chair, 3(2), 167-178. 


\section{Anexos}

Tabla 1. Transcripción de la escena.

\begin{tabular}{|c|c|c|}
\hline Escena & Nombre & Discurso \\
\hline 1. & $\mathrm{~T}$ & $\begin{array}{l}\text { En la cuadrícula siguiente se han construido los triángulos de color } \\
\text { rojo, verde y azul... la cuadrícula está conformada por cuadros de un } \\
\text { centímetro de lado como se muestra en la figura... ya pues, ustedes } \\
\text { tienen sus dibujos... los triángulos rojo, verde y azul... y nos } \\
\text { preguntan: analiza y responde ¿qué triángulo tiene más área?, } \\
\text { argumenta tu respuesta seleccionada, entonces la primera opción } \\
\text { es: el área del triángulo rojo, el área del triángulo verde, el área del } \\
\text { triángulo azul o ninguno de los anteriores, o todos miden igual. } \\
\text { Ahora a mi amigo Víctor le voy a preguntar: ¿qué información de la } \\
\text { que leíste te ayudó para que tú respondieras...... los demás van } \\
\text { pensando la respuesta... }\end{array}$ \\
\hline 2. & Sam & Los cuadros que tiene la medida para sacar el área \\
\hline 3. & $\mathrm{~T}$ & Y ¿̇cuánto es su medida? \\
\hline 4. & Sam & $1 \mathrm{~cm}$ \\
\hline 5. & $\mathrm{~T}$ & Ok... \\
\hline 6. & Erick & $\begin{array}{l}\text { Los cuadrados de } 1 \mathrm{~cm} \text { que me ayudaron a sacar el área de todos } \\
\text { los triángulos }\end{array}$ \\
\hline 7. & $\mathrm{~T}$ & Listo \\
\hline 8. & Álvaro & Los cuadros que miden $1 \mathrm{~cm} . .$. \\
\hline 9. & $\mathrm{~T}$ & ¿Qué información te ayudó del problema?... \\
\hline 10. & Ingrid & Los cuadros... \\
\hline 11. & Itzel & Los cuadros... \\
\hline 12. & $\mathrm{~T}$ & ¿Algo en especial de los cuadros?... \\
\hline 13. & Itzel & Las líneas... \\
\hline 14. & $\mathrm{~T}$ & Mi amigo Víctor ¿qué te ayudó? \\
\hline 15. & Víctor & Los cuadros y los tres triángulos; rojo verde y azul... \\
\hline 16. & $\mathrm{~T}$ & Ok... \\
\hline 17. & $\ldots$ & $\ldots$ \\
\hline
\end{tabular}




\begin{tabular}{|c|c|c|}
\hline 18. & $\mathrm{~T}$ & $\begin{array}{l}\text { Mi amigo Víctor, ayúdame, ¿cuál es tu respuesta a la pregunta?... Y } \\
\text { me vas a decir ¿por qué? }\end{array}$ \\
\hline 19. & Víctor & El área del triángulo azul... \\
\hline 20. & $\mathrm{~T}$ & El área del triángulo azul es ¿queé? o ¿más qué? \\
\hline 21. & Víctor & $\begin{array}{l}\text { El azul tiene más base que el rojo y el verde y se saca más rápido } \\
\text { la base del azul [...] }\end{array}$ \\
\hline 22. & $\mathrm{~T}$ & Ok... \\
\hline 23. & $\mathrm{~T}$ & Tú levantaste la mano, ¿̇qué puedes decir de la respuesta de Víctor? \\
\hline 24. & Álvaro & Que todos los triángulos tienen la misma área... \\
\hline 25. & $\mathrm{~T}$ & $\begin{array}{l}\text { Tú dices que todos tienen la misma área, escuchen... tú dijiste que } \\
\text { no [iNo! dijo Víctor en voz baja]... ¿̇Por qué dijiste que no? }\end{array}$ \\
\hline 26. & Víctor & Porque el rojo tiene $9 \mathrm{~cm}$ de base, el verde y el azul tienen $12 \mathrm{~cm}$... \\
\hline 27. & $\mathrm{~T}$ & ¿Y entonces...? \\
\hline 28. & Víctor & Son más... tienen más base el verde y el azul \\
\hline 29. & $\mathrm{~T}$ & Y ¿qué pasa si tienen más base el verde y el azul? \\
\hline 30. & & $\ldots$ \\
\hline 31. & Víctor & Se saca más rápido... [el área de los triángulos] \\
\hline 32. & $\mathrm{~T}$ & Ok... \\
\hline 33. & $\mathrm{~T}$ & Ahora mi amigo Samuel... \\
\hline 34. & Sam & $\begin{array}{l}\text { El resultado es que todos tienen la misma área porque aunque } \\
\text { tenga } 9 \text { aumenta a más la altura [se refiere a la altura del triángulo } \\
\text { rojo]... y cuando multiplicas te da } 36 \text { y dividido entre dos te da } 18 . . .\end{array}$ \\
\hline 35. & $\mathrm{~T}$ & ¿Cómo sabes tú que el área de todos esos triángulos son iguales? \\
\hline 36. & Sam & Porque saqué el área... la fórmula... \\
\hline 37. & $\mathrm{~T}$ & Porque sacaste la fórmula del... \\
\hline 38. & Sam & De todos los triángulos... \\
\hline 39. & $\mathrm{~T}$ & ¿Y cuánto te dio el área de todos los triángulos...? \\
\hline 40. & Sam & El del azul me dio 36 , el verde 36 y el rojo $36 \ldots$ \\
\hline 41. & $\mathrm{~T}$ & OK... \\
\hline 42. & $\mathrm{~T}$ & $\begin{array}{l}\text { Mi amigo Erick... ¿qué puedes decir de la respuesta que te dijo tu } \\
\text { amigo Samuel? }\end{array}$ \\
\hline
\end{tabular}




\begin{tabular}{|c|c|c|}
\hline 43. & Erick & $\begin{array}{l}\text { Todos tienen la misma área porque... aunque el rojo tenga menos } \\
\text { base también le ayuda la altura... }\end{array}$ \\
\hline 44. & $\mathrm{~T}$ & ¿Pero cómo le ayuda la altura? \\
\hline 45. & Erick & Lo multipliqué base por altura... \\
\hline 46. & $\mathrm{~T}$ & ¿Y cuánto te da? \\
\hline 47. & Erick & $\begin{array}{l}\text { Base por altura sobre dos; el primero, el triángulo rojo me da } 9 \text { por } \\
4 \text { igual a } 36 . . .\end{array}$ \\
\hline 48. & $\mathrm{~T}$ & ¿Después...? \\
\hline 49. & Erick & 36 entre dos da 18 \\
\hline 50. & $\mathrm{~T}$ & ¿Después qué haces...? \\
\hline 51. & Erick & Y el otro me da 18 también... \\
\hline 52. & $\mathrm{~T}$ & ¿Qué me ibas a decir tú?, discúlpame... \\
\hline 53. & Álvaro & $\begin{array}{l}\text { Que los tres son iguales [se refiere a la medida de las áreas de los } \\
\text { triángulos] porque tienen la misma base y altura, y cuando se } \\
\text { multiplica la base y la altura de los tres triángulos... }\end{array}$ \\
\hline 54. & $\mathrm{~T}$ & ¿Pero solo multiplicas y ya? \\
\hline 55. & Álvaro & Y los divide... \\
\hline 56. & $\mathrm{~T}$ & ¿Y te da lo mismo verdad...? \\
\hline 57. & Álvaro & Ajá... \\
\hline 58. & $\mathrm{~T}$ & Ok \\
\hline 59. & $\mathrm{~T}$ & Vamos a escuchar a mi amiga Itzel... ¿cuál fue tu respuesta? \\
\hline 60. & Itzel & El triángulo azul \\
\hline 61. & $\mathrm{~T}$ & Tienes más área... ¿̇por qué? \\
\hline 62. & Itzel & Porque tiene más cuadrados \\
\hline 63. & $\mathrm{~T}$ & ¿Pero en dónde tiene más cuadros? \\
\hline 64. & Itzel & Tiene más base el triángulo azul y el verde que el rojo... \\
\hline 65. & $\mathrm{~T}$ & ¿Tú qué dices mi amigo Álvaro? \\
\hline 66. & Álvaro & Pero acá está diciendo que tenemos que sacar el área, no la base... \\
\hline 67. & $\mathrm{~T}$ & $\begin{array}{l}\text { Mi amigo Álvaro dice que no solo debemos contar la base ni la } \\
\text { altura nada más... si no que debemos sacar el área }\end{array}$ \\
\hline 68. & Erick & Es el resultado de todos... [ el área de los triángulos] \\
\hline
\end{tabular}




\begin{tabular}{|c|c|c|}
\hline 69. & Sam & $\begin{array}{l}\text { Aunque la base del triángulo azul, son iguales [las áreas]... porque } \\
\text { dan la misma... }\end{array}$ \\
\hline 70. & $\mathrm{~T}$ & ¿Dan la misma qué? \\
\hline 71. & Sam & El mismo resultado \\
\hline 72. & $\mathrm{~T}$ & Víctor defiende lo que hiciste \\
\hline 73. & Victor & El azul tenía más base que los otros \\
\hline 74. & $\mathrm{~T}$ & Más base y ¿̇qué más? \\
\hline 75. & Víctor & Se puede sacar más rápido [el área del triángulo azul] \\
\hline 76. & $\mathrm{~T}$ & ¿Cuánto es el área del triángulo azul? \\
\hline 77. & Victor & $12 \mathrm{~cm}$ por la altura $3 \mathrm{~cm}$ entre dos, y me dio... 18 \\
\hline 78. & $\mathrm{~T}$ & Y ¿̇uánto es el área del triángulo verde? \\
\hline 79. & Víctor & 18 \\
\hline 80 & $\mathrm{~T}$ & Y ¿̇cuánto es el área del triángulo rojo? \\
\hline 81. & Víctor & 18 \\
\hline 82. & $\mathrm{~T}$ & Entonces ¿por qué tu respuesta es el triángulo azul? \\
\hline 83. & & $\ldots$ \\
\hline 84. & $\mathrm{~T}$ & ¿Qué dice mi amiga Ingrid? ¿Cuál fue tu respuesta? \\
\hline 85. & Ingrid & Azul \\
\hline 86. & $\mathrm{~T}$ & ¿Por qué? Defiende tu respuesta \\
\hline 87. & Ingrid & Porque el azul se ve más grande \\
\hline 88. & $\mathrm{~T}$ & ¿El azul no se ve más grande?... \\
\hline 89. & Víctor & $\begin{array}{l}\text { Pero no importa que esté más grande, lo que importa es que se } \\
\text { multiplique su base por su altura [y] midan lo mismo }\end{array}$ \\
\hline 90. & Sam & $\begin{array}{l}\text { Mientras que te dé lo mismo no importa que sea más grande... } \\
\text { importa el resultado... }\end{array}$ \\
\hline 91. & Álvaro & Lo mismo... \\
\hline 92. & $\mathrm{~T}$ & Ingrid defiende ¿qué dices tú? \\
\hline 93. & Ingrid & Es que el triángulo azul tiene más base y los otros menos... \\
\hline 94. & Álvaro & $\begin{array}{l}\text { Es que no importa la medida de la base, importa todo... la altura, la } \\
\text { base y [que] al multiplicarlo y dividirlo den lo mismo. }\end{array}$ \\
\hline
\end{tabular}


